\title{
Temporally multiplexed superposition states of continuous variables
}

\author{
Masahide Sasaki, ${ }^{1,2}$ Masahiro Takeoka, ${ }^{1,2}$ and Hiroki Takahashi ${ }^{1,3,2}$ \\ 1 National Institute of Information and Communications Technology, \\ 4-2-1 Nukui-Kita, Koganei, Tokyo 184-8795, Japan \\ 2 CREST, Japan Science and Technology Agency, \\ 5 Sanbanchuoh, Chiyoda-ku, Tokyo 102-0075, Japan \\ 3 Department of Applied Physics, The University of Tokyo 7-3-1 Hongo, Bunkyo-ku, Tokyo 113-8656, Japan
}

(Dated: August 9, 2021)

\begin{abstract}
We study non-Gaussian states generated by two-photon subtraction from a cw squeezed light source. In a cw scheme one can subtract two photons from the source with a designated time separation and can genarate temporally multiplexed superposition states of continuous variables. We numerically study the properties of these states in the light of bosonic interference in the time domain. In an appropriate temporal mode amplified kittens are produced in a region where the time separation is comparable with the correlation time of squeezed packets.
\end{abstract}

PACS numbers:

\section{INTRODUCTION}

In optical quantum information processing there have been two kinds of approaches with discrete-variable (DV) and continuous-variable (CV) schemes. The former usually consists of entangled photon states and photon counters, while the latter consists of squeezed states and homodyne detectors. Each of these schemes is, however, only a part of control with full potential aspects of quantum field of light. The scheme combining these two will surely be promissing for exploiting universal quantum information processing for computing, communications, and metrology.

Recent progress in this direction has been made as the generation of quantum superposition of mesoscopically distinguishable states using a squeezed state, photon counting, and homodyne detection [1, 2, 3, 4, 5]. These experiments are based on the photon subtraction from a squeezed state, as proposed by Dakna et al. [6]. In the scheme a small fraction of squeezed light is tapped via a beam splitter, and is guided into photon counters. The remaining light beam conditioned on photon clicks turns to be a highly nonclassical and non-Gaussian state, which was transformed from a squeezed state (Gaussian state) via a strong nonlinear process of photon counting. In this way quantum superposition of mesoscopically distinguishable states (Schrödinger kitten states) were generated by the single-photon subtraction.

Next step is to produce two or more kitten states as well as to increase the size of cat states for realizing quantum information processing and quantum metrology [7, 8, 9]. If larger number of photons could be subtracted from a squeezed light, larger size of quantum superpositions (Schrödinger cat states) could be produced, although experiments get more challenging [10]. Another method is to produce a larger cat state from two smaller cat states as proposed in [11]. A new method to produce larger cat states was recently demonstrated in laboratory, by using two-photon Fock state with conditional homodyning [12].
In this paper, we study another avenue in this direction, which is the generation of two kitten states with a designated time separation in the temporal domain by using the two-photon subtraction from a cw squeezed light. Two kitten states are generated in a single spatial mode. They are of temporally two-mode, and generally entangled, referred to as temporally multiplexed kitten states. We numerically study the properties of these states in the light of bosonic interference in the time domain, and show that in an appropriate temporal mode amplified kittens are produced in a region where the time separation is comparable with the correlation time of squeezed packets. The paper is organized as follows. In Sec. II we introduce basic notions for our cw scheme, and provide a rough sketch of physics with an idealized lossless model. In Sec. III we present a precise modeling in a practical setting, show numerical results, and discuss bosonic interference occuring in the cross over region where the time separation is comparable with the correlation time of squeezed packets. Section IV concludes the paper.

\section{PHOTON SUBTRACTION IN A CW SCHEME: ROUGH SKETCH OF PHYSICS}

The photon subtraction scheme is depicted in Fig. 1 A small fraction of the squeezed beam in path $\mathrm{A}$ is tapped at a beam splitter (BS) with reflectance $R$, guided into two photodetectors in paths $\mathrm{B}$ and $\mathrm{C}$ through an optical filter and another beam splitter with reflectance $R_{1}$, and is then used as trigger signals for conditional photon subtraction.

We consider the scheme with a cw squeezed light generated by an optical parametric oscillator (OPO), as studied extensively by Nielsen and Mølmer [13, 14, 15]. A typical feature of this scheme is that the trigger photodetection takes place in much shorter time scale $(<1 \mathrm{~ns}$ for Si APD) compared with the OPO time scale ( 100 ns), and hence interesting multimode kitten states can be generated in the time domain [2, 3]. This is in sharp contrast 


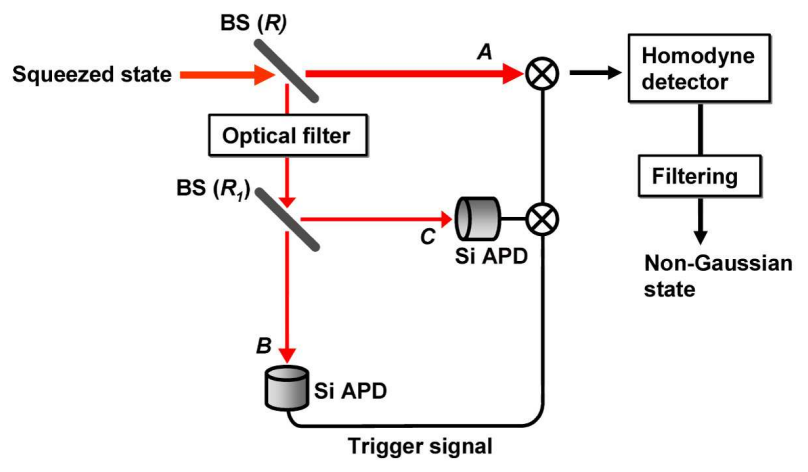

FIG. 1: (Color online) Scheme of the two-photon subtraction from a squeezed state. BSs are beam splitters with the reflectances of $R$ and $R_{1}$.

to the short pulse scheme where the photon counting time scale is longer than the pulse width [1, [4, [5, 12].

The annihilation field operator in a $\mathrm{cw}$ scheme is denoted by

$$
\hat{a}(t)=\frac{1}{2 \pi} \int_{-\infty}^{\infty} d \Omega \hat{a}\left(\omega_{0}+\Omega\right) e^{-i\left(\omega_{0}+\Omega\right) t} .
$$

where $\omega_{0}$ is the center angular frequency of the spectrum of a laser source. The operator obeys the continuum commutation relation

$$
\left[\hat{a}\left(\omega_{0}+\Omega\right), \hat{a}^{\dagger}\left(\omega_{0}+\Omega^{\prime}\right)\right]=2 \pi \delta\left(\Omega-\Omega^{\prime}\right) .
$$

The time dependent field operator $\hat{a}(t)$ is defined in the interval $(-\infty, \infty)$, and obeys the commutation relation

$$
\left[\hat{a}(t), \hat{a}^{\dagger}\left(t^{\prime}\right)\right]=\delta\left(t-t^{\prime}\right)
$$

Squeezed light fields can be conveniently described in the rotating frame about the center frequency $\omega_{0}$,

$$
\hat{A}(t)=\hat{a}(t) e^{i \omega_{0} t}=\frac{1}{2 \pi} \int_{-\infty}^{\infty} d \Omega \hat{A}(\Omega) e^{-i \Omega t}
$$

where $\hat{A}(\Omega)=\hat{a}\left(\omega_{0}+\Omega\right)$.

Now let us roughly sketch an essential physics by assuming a nearly lossless OPO squeezing described by the Bogolubov transformation

$$
\hat{S}_{A}^{\dagger} \hat{A}(\Omega) \hat{S}_{A}=\mu(\Omega) \hat{A}(\Omega)+\nu(\Omega) \hat{A}^{\dagger}(-\Omega) .
$$

The cw beam described by a pure state $\hat{S}_{A}\left|\mathbf{0}_{A}\right\rangle$ is the input squeezed state for photon subtraction.

\section{A. Odd-number Schrödinger kitten by single-photon subtraction}

Let us first consider the single-photon subtraction with a trigger signal detected at $t=t_{1}$ in the detector in path $\mathrm{B}\left(R_{1}=0\right.$ in Fig. 1). We assume that this detection takes place instantaneously compared with the OPO time scale, and the trigger beam is projected onto the vacua for other times. Denoting the BS operation with the reflectance $R$ as $\hat{V}_{A B}$, the conditional (not normalized) state is then described by

$$
\begin{aligned}
\left|\rho_{C W}^{(1)}\right\rangle & \propto\left\langle\mathbf{0}_{B}\left|\hat{B}\left(t_{1}\right) \hat{V}_{A B} \hat{S}_{A}\right| \mathbf{0}_{A B}\right\rangle \\
& =-\sqrt{R} \hat{A}\left(t_{1}\right) \hat{S}_{A}\left|\mathbf{0}_{A}\right\rangle \\
& =-\sqrt{R} \hat{S}_{A} \int_{-\infty}^{\infty} d t \hat{A}^{\dagger}(t) \nu\left(t-t_{1}\right)\left|\mathbf{0}_{A}\right\rangle
\end{aligned}
$$

where we have used the relation (5), and introduced

$$
\nu\left(t-t_{1}\right) \equiv \frac{1}{2 \pi} \int_{-\infty}^{\infty} d \Omega e^{i \Omega\left(t-t_{1}\right)} \nu(\Omega) .
$$

We have also assumed that $R$ is small so that the renormalization of the effective squeezing on beam $\mathrm{A}$ due to optical loss is negligible.

This quantity represents the temporal correlation of squeezed photons. It is roughly given by

$$
\nu(t) \sim \frac{\epsilon}{\sqrt{\zeta_{0}}} \psi(t)
$$

where

$$
\psi(t)=\sqrt{\zeta_{0}} e^{-\zeta_{0}|t|}
$$

is a normalized temporal mode function [13, 14, 15]. Here $\zeta_{0} / \pi$ corresponds to the OPO resonant bandwidth, and $\epsilon$ is the nonlinear coefficient of the OPO, propotional to the $\chi^{(2)}$ interaction coefficient and the pump field amplitude. Defining the field operator localized in this temporal mode by

$$
\hat{A}_{1}^{\dagger}=\int_{-\infty}^{\infty} d t \hat{A}^{\dagger}(t) \psi\left(t-t_{1}\right)
$$

the single-photon subtracted state can be written as

$$
\left|\rho_{C W}^{(1)}\right\rangle \propto-\frac{\sqrt{R} \epsilon}{\sqrt{\zeta_{0}}} \hat{S}_{A} \hat{A}_{1}^{\dagger}\left|\mathbf{0}_{A}\right\rangle
$$

This represents a $\mathrm{cw}$ beam consisting of the squeezed single-photon state [11, 16] localized in the mode $\psi\left(t-t_{1}\right)$ and the squeezed vacua in the other time domains.

The beam is filtered through the mode function $\psi(t-$ $t_{1}$ ), and then sampled by homodyne detection [13]. In the homodyne output one can see that two distinguishable waveforms with phase difference of $\pi$ coexist as demonstrated experimentally in [1, 2, 3]. The constructed Wigner functions have negative part, showing the nonclassicality of the states. This is a quantum superposition of mesoscopically distinguishable states as analyzed by Dakna et al. [6], the so called Schrödinger kitten states. In this case it is an odd-number kittens. 


\section{B. Entangled kittens by two-photon subtraction}

In the two-photon subtraction, we set $R_{1}=0.5$ (i.e. $50 \%$ ) in Fig. 1. Suppose that a first and second photon are detected at $t=t_{1}$ in path $\mathrm{B}$, and at $t=t_{2}$ in path $\mathrm{C}$, respectively. The conditional state can be represented as

$$
\begin{aligned}
& \left|\rho_{C W}^{(2)}\right\rangle \\
& \propto\left\langle\mathbf{0}_{B C}\left|\hat{C}\left(t_{2}\right) \hat{B}\left(t_{1}\right) \hat{V}_{B C} \hat{V}_{A B} \hat{S}_{A}\right| \mathbf{0}_{A B C}\right\rangle \\
& =\frac{R}{2} \hat{A}\left(t_{2}\right) \hat{A}\left(t_{1}\right) \hat{S}_{A}\left|\mathbf{0}_{A}\right\rangle \\
& =\frac{R}{2} \hat{S}_{A}\left[\int_{-\infty}^{\infty} d t^{\prime} \hat{A}^{\dagger}\left(t^{\prime}\right) \nu\left(t^{\prime}-t_{2}\right) \int_{-\infty}^{\infty} d t \hat{A}^{\dagger}(t) \nu\left(t-t_{1}\right)\right. \\
& \left.+\int_{-\infty}^{\infty} \frac{d \Omega}{2 \pi} e^{i \Omega\left(t_{2}-t_{1}\right)} \mu(-\Omega) \nu(\Omega)\right]\left|\mathbf{0}_{A}\right\rangle \\
& \sim \frac{R}{2} \hat{S}_{A}\left[\frac{\epsilon^{2}}{\zeta_{0}} \hat{A}_{2}^{\dagger} \hat{A}_{1}^{\dagger}+\frac{\epsilon}{\sqrt{\zeta_{0}}} \psi\left(t_{2}-t_{1}\right)\right]\left|\mathbf{0}_{A}\right\rangle \text {. }
\end{aligned}
$$

One can immediately see the two extremes;

(i) $\Delta\left(\equiv\left|t_{2}-t_{1}\right|\right) \ll \zeta_{0}^{-1}$ where the two-photon subtraction takes place in the single mode, producing an even-number kitten as originally proposed in [6].

(ii) $\Delta \gg \zeta_{0}^{-1}$ where two odd-number kittens are generated in separated packets $\psi\left(t-t_{1}\right)$ and $\psi\left(t-t_{2}\right)$. Each kitten is the one generated in [2, 3].

In the imtermediate region there is a cross over between (i) and (ii), and both even- and odd-kittens exsist, being entangled over the two modes. This region is of our interest.

Since the field operators $\hat{A}_{1}^{\dagger}$ and $\hat{A}_{2}^{\dagger}$ have generally a finite mode overlap for finite $\Delta$

$$
\begin{aligned}
I_{\Delta} & \equiv\left\langle\mathbf{0}_{A}\left|\hat{A}_{2} \hat{A}_{1}^{\dagger}\right| \mathbf{0}_{A}\right\rangle \\
& =\int_{-\infty}^{\infty} d t \psi\left(t-t_{1}\right) \psi\left(t-t_{2}\right) \\
& =\left(1+\zeta_{0} \Delta\right) e^{-\zeta_{0} \Delta}
\end{aligned}
$$

one needs an orthonormal mode set to describe the state.

\section{Unbiased modes}

We first consider an orthonormal mode function pair $\left\{\Psi_{U 1}(t), \Psi_{U 2}(t)\right\}$ which suits to analyze the region $\zeta_{0} \Delta>$ 1 , and defined by

$$
\begin{aligned}
& \psi\left(t-t_{1}\right)=\sqrt{1-p_{\Delta}} \Psi_{U 1}(t)+\sqrt{p_{\Delta}} \Psi_{U 2}(t) \\
& \psi\left(t-t_{2}\right)=\sqrt{p_{\Delta}} \Psi_{U 1}(t)+\sqrt{1-p_{\Delta}} \Psi_{U 2}(t)
\end{aligned}
$$

where

$$
p_{\Delta}=\frac{1-\sqrt{1-I_{\Delta}^{2}}}{2} .
$$

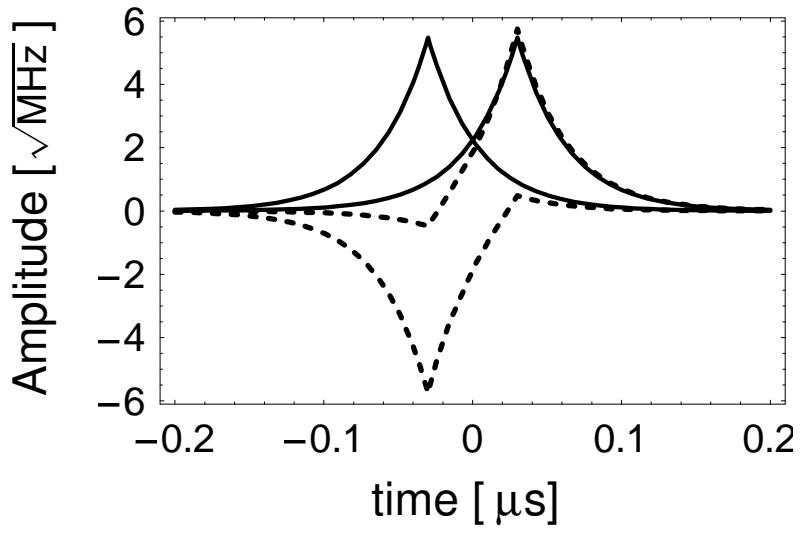

FIG. 2: Temporal shape of the unbiased modes (dashed lines) in the case of $\zeta_{0}=30 \mathrm{MHz}$. The solid lines are $\psi\left(t-t_{1}\right)$ and $\psi\left(t-t_{2}\right)$.

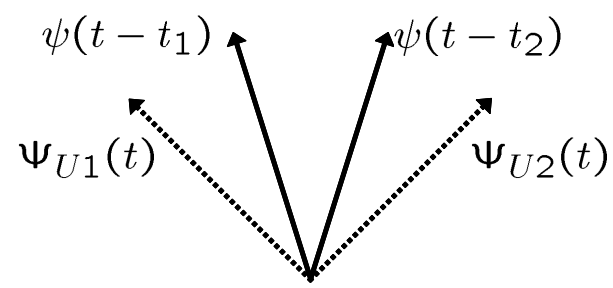

FIG. 3: Vector representation of the unbiased modes.

We call them the unbiased modes because $\Psi_{U 1}(t)$ and $\Psi_{U 2}(t)$ equally overlap with $\psi\left(t-t_{1}\right)$ and $\psi\left(t-t_{2}\right)$, respectively. Their temporal shapes are shown in Fig. 2 as well as the mode overlap configuration in vector representation in Fig. 3. The corresponding field operators are defined by

$$
\hat{A}_{U i} \equiv \int_{-\infty}^{\infty} d t \hat{A}(t) \Psi_{U i}(t)
$$

satisfying

$$
\left[\hat{A}_{U i}, \hat{A}_{U j}^{\dagger}\right]=\delta_{i j}
$$

The operators $\hat{A}_{i}^{\dagger}$ are represented as

$$
\begin{aligned}
& \hat{A}_{1}^{\dagger}=\sqrt{1-p_{\Delta}} \hat{A}_{U 1}^{\dagger}+\sqrt{p_{\Delta}} \hat{A}_{U 2}^{\dagger} \\
& \hat{A}_{2}^{\dagger}=\sqrt{p_{\Delta}} \hat{A}_{U 1}^{\dagger}+\sqrt{1-p_{\Delta}} \hat{A}_{U 2}^{\dagger} .
\end{aligned}
$$

The two-photon subtracted state (not noralized) is then represented as

$$
\begin{aligned}
& \hat{A}\left(t_{2}\right) \hat{A}\left(t_{1}\right) \hat{S}_{A}\left|\mathbf{0}_{A}\right\rangle \\
& \sim \hat{S}_{A}\left[\frac{\epsilon^{2}}{\zeta_{0}}\left(|1,1\rangle+I_{\Delta} \frac{|2,0\rangle+|0,2\rangle}{\sqrt{2}}\right)\right. \\
& \left.\quad+\epsilon e^{-\zeta_{0} \Delta}|0,0\rangle\right]\left|\mathbf{0}_{\tilde{A}}\right\rangle .
\end{aligned}
$$


The ket vectors in the above equation are understood as $|\cdots, \cdots\rangle=|\cdots\rangle_{U 1} \otimes|\cdots\rangle_{U 2}$. Thus the state in terms of the unbiased modes is invariant under the mode permutation. The $\Delta$-dependence of the above state is completely governed by the coefficients in the bracket. Note that $\hat{S}_{A}$ is independent of $\Delta$. The index $\tilde{A}$ means the other remaining modes than those spanned by the $U 1$ and the $U 2$. The modes $\tilde{A}$ will be filtered out to observe the two-photon subtracted states.

The two-mode state is essentially an entangled state of the two kinds of kittens (the odd-number and evennumber kittens), and the squeezed vacuum. In fact the two-photon subtracted state can also be represented as

$$
\begin{aligned}
&\left|\rho_{C W}^{(2)}\right\rangle \equiv \frac{\hat{A}\left(t_{2}\right) \hat{A}\left(t_{1}\right) \hat{S}_{A}\left|\mathbf{0}_{A}\right\rangle}{\| \hat{A}\left(t_{2}\right) \hat{A}\left(t_{1}\right) \hat{S}_{A}\left|\mathbf{0}_{A}\right\rangle \|} \\
& \sim \mathcal{N} \hat{S}_{A}\left[\frac{\epsilon}{\zeta_{0}}|1,1\rangle\right. \\
& \\
&\left.+e^{-\zeta_{0} \Delta} \nu_{e}\left(\left|\phi_{e}, 0\right\rangle+\left|0, \phi_{e}\right\rangle\right)\right]\left|\mathbf{0}_{\tilde{A}}\right\rangle
\end{aligned}
$$

where

$$
\begin{aligned}
\left|\phi_{e}\right\rangle & \equiv \nu_{e}^{-1}\left[\frac{\epsilon}{\zeta_{0}}\left(1+\zeta_{0} \Delta\right)|2\rangle+\frac{1}{\sqrt{2}}|0\rangle\right] \\
\mathcal{N} & \equiv\left[\frac{\epsilon^{2}}{\zeta_{0}^{2}}+e^{-2 \zeta_{0} \Delta}\left(\nu_{e}^{2}+\frac{1}{2}\right)\right]^{-1 / 2} \\
\nu_{e} & \equiv \sqrt{\frac{\epsilon^{2}}{\zeta_{0}^{2}}\left(1+\zeta_{0} \Delta\right)^{2}+\frac{1}{2}}
\end{aligned}
$$

The first term includes two odd-number kittens in the similar sense of Eq. (11). As for the second term, when $\left|\phi_{e}\right\rangle$ is squeezed, one has an even-number kitten as demonstrated by Dakna et al. [6]. (Note that this is not precisely a coherent-state superposition but a superposition of other kinds of two mesoscopically distinct components with even-number photons. The detailed features will be presented in the next section numerically.)

The characteristic features are summarized as follows:

(i) The first term in Eq. (20) includes the two singlephoton-state kernels, each comes from the distinguishable photon subtraction from each squeezed light packet in $\psi\left(t-t_{1}\right)$ and $\psi\left(t-t_{2}\right)$. This corresponds to the two odd-number kittens. (See Eq. (11).)

(ii) The second and third terms with $\left|\phi_{e}\right\rangle$ in Eq. (20) include the two even-number-state kernels. They come from the indistinguishable photon subtraction from the overlapping components between the two localized packets in $\psi\left(t-t_{1}\right)$ and $\psi\left(t-t_{2}\right)$, and their weights decreases as $\Delta$. The $\left|\phi_{e}\right\rangle$ being squeezed, the resulting state is an even-number kitten.

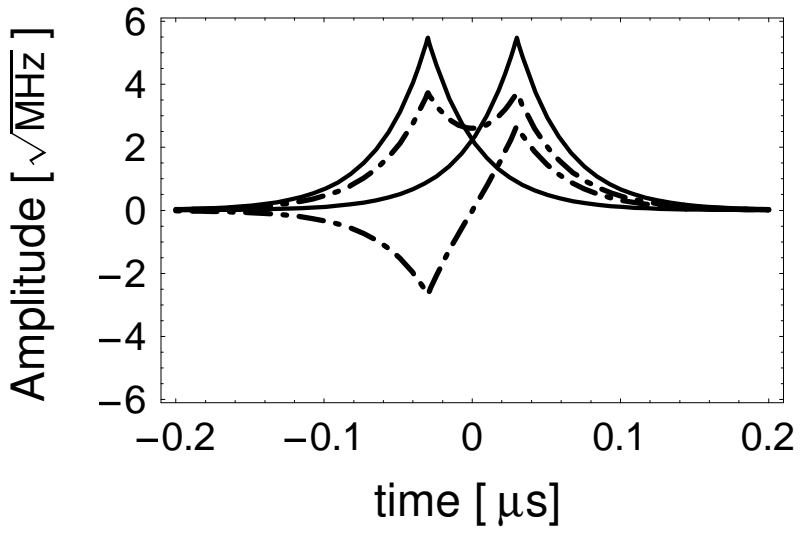

FIG. 4: Temporal shape of the biased modes (one-dotted lines) in the case of $\zeta_{0}=30 \mathrm{MHz}$. The $\Psi_{+}(t)$ is symmetric, while the $\Psi_{-}(t)$ is asymmetric. The solid lines are $\psi\left(t-t_{1}\right)$ and $\psi\left(t-t_{2}\right)$.

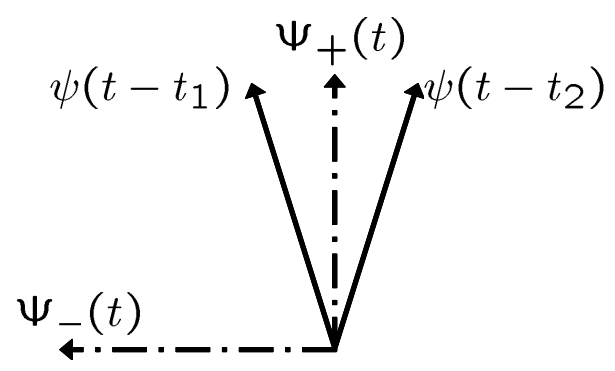

FIG. 5: Vector representation of the unbiased modes.

\section{Biased modes}

Let us then consider the other important mode function pair, which suits to analyze the region $\zeta_{0} \Delta<1$, and defined by

$$
\Psi_{ \pm}(t)=\frac{\psi\left(t-t_{2}\right) \pm \psi\left(t-t_{1}\right)}{\sqrt{2\left(1 \pm I_{\Delta}\right)}}
$$

Their temporal shapes are shown in Fig. 4 as well as the mode overlap configuration in vector representation in Fig. 5. The $\Psi_{+}(t)$ is the mode that extracts the features due to the overlap of the packets $\psi\left(t-t_{1}\right)$ and $\psi\left(t-t_{2}\right)$, while the $\Psi_{-}(t)$ is the mode that extracts the differential features of these localized packets. We refer them to as the biased modes. Defining the field operators in the biased modes by

$$
\hat{A}_{ \pm} \equiv \int_{-\infty}^{\infty} d t \hat{A}(t) \Psi_{ \pm}(t)
$$

and the number states by $\left|n_{+}\right\rangle=\frac{\hat{A}_{+}^{\dagger n}}{\sqrt{n !}}\left|0_{+}\right\rangle$the twophoton subtracted state can either be represented as

$$
\hat{A}\left(t_{2}\right) \hat{A}\left(t_{1}\right) \hat{S}_{A}\left|\mathbf{0}_{A}\right\rangle
$$




$$
\begin{aligned}
\sim \hat{S}_{A}[ & \frac{\epsilon^{2}}{\zeta_{0}}\left(\frac{1+I_{\Delta}}{\sqrt{2}}\left|2_{+}, 0_{-}\right\rangle-\frac{1-I_{\Delta}}{\sqrt{2}}\left|0_{+}, 2_{-}\right\rangle\right) \\
& \left.+\epsilon e^{-\zeta_{0} \Delta}\left|0_{+}, 0_{-}\right\rangle\right]\left|\mathbf{0}_{\tilde{A}}\right\rangle .
\end{aligned}
$$

Thus in the biased mode the output state is a squeezed entangled-state of 2-photon and the vacuum states over modes $\Psi_{ \pm}(t)$. This is actually an entangled state of evennumber kitten state and the squeezed vacuum state. For $\zeta_{0} \Delta \ll 1$

$$
\begin{aligned}
\hat{A}\left(t_{2}\right) \hat{A}\left(t_{1}\right) \hat{S}_{A}\left|\mathbf{0}_{A}\right\rangle \rightarrow & \hat{S}_{A+}\left(\sqrt{2} \frac{\epsilon^{2}}{\zeta_{0}}\left|2_{+}\right\rangle+\epsilon\left|0_{+}\right\rangle\right) \\
& \otimes \hat{S}_{A-}\left|0_{-}\right\rangle \otimes \hat{S}_{\tilde{A}}\left|\mathbf{0}_{\tilde{A}}\right\rangle
\end{aligned}
$$

where we have approximately decompose the squeezing operator $\hat{S}_{A}$ into a product of the operators for the modes $\Psi_{+}(t), \Psi_{-}(t)$ and others. Most of photons are contained in mode $\Psi_{+}(t)$ while mode $\Psi_{-}(t)$ is nearly the vacuum state. Actually the squeezing in the aymmetric mode $\Psi_{-}(t)$ is very small. This feature holds for $\zeta_{0} \Delta \lesssim 1$, and for certain finite $\Delta$ 's, an amplified even-number cat state is produced in mode $\Psi_{+}(t)$ as shown in the next section.

For $\zeta_{0} \Delta \gg 1$ on the other hand photons are distributed equally to modes $\Psi_{+}(t)$ and $\Psi_{-}(t)$ as

$$
\begin{aligned}
& \hat{A}\left(t_{2}\right) \hat{A}\left(t_{1}\right) \hat{S}_{A}\left|\mathbf{0}_{A}\right\rangle \\
& \rightarrow \frac{\epsilon^{2}}{\sqrt{2} \zeta_{0}} \hat{S}_{A+} \otimes \hat{S}_{A-}\left(\left|2_{+}, 0_{-}\right\rangle-\left|0_{+}, 2_{-}\right\rangle\right) \\
& \quad \otimes \hat{S}_{\tilde{A}}\left|\mathbf{0}_{\tilde{A}}\right\rangle
\end{aligned}
$$

Thus the two-photon subtraction from a cw squeezed beam generates temporally multiplexed Schrödinger kittens over the two temporal modes. In the next section we show the features described above by calculating the Wigner functions based on the precise modeling in a practical setting. A new formalism to treat only the two modes of interest with the effective super-operators and detailed analysis of the states are presented elswhere [19].

\section{PHOTON SUBTRACTION IN A CW SCHEME: PRECISE MODELING}

\section{A. Model}

In this section we extend the idealized lossless model described above to a practical case, and provide the formulae and numerical results for future experiments. In a practical setting of OPO, the pure-state argument in the previous section cannot apply because the two temporal modes have generally quantum correlations with the other modes (other neighboring squeezed packets). This makes the relevant states mixed more or less. One needs to calculate density operators, whose details are given in Appendix.
A cw squeezed state generated by an OPO, $\hat{\rho}_{A}^{(0)}$, is fully characterized in the time domain by the correlation functions [17]

$$
\begin{aligned}
& \operatorname{Tr}\left[\hat{\rho}_{A}^{(0)} \hat{A}^{\dagger}(t) \hat{A}\left(t^{\prime}\right)\right] \\
& =\frac{\epsilon \gamma_{T}}{4}\left(\frac{e^{-\zeta(-\epsilon)\left|t-t^{\prime}\right|}}{\zeta(-\epsilon)}-\frac{e^{-\zeta(\epsilon)\left|t-t^{\prime}\right|}}{\zeta(\epsilon)}\right) \\
& \operatorname{Tr}\left[\hat{\rho}_{A}^{(0)} \hat{A}(t) \hat{A}\left(t^{\prime}\right)\right] \\
& =\frac{\epsilon \gamma_{T}}{4}\left(\frac{e^{-\zeta(-\epsilon)\left|t-t^{\prime}\right|}}{\zeta(-\epsilon)}+\frac{e^{-\zeta(\epsilon)\left|t-t^{\prime}\right|}}{\zeta(\epsilon)}\right)
\end{aligned}
$$

where

$$
\zeta(\epsilon) \equiv \zeta_{0}+\epsilon, \quad \zeta_{0} \equiv \frac{\gamma_{T}+\gamma_{L}}{2}
$$

with the leakage rates $\gamma_{T}$ of the output coupler and $\gamma_{L}$ of the cavity loss. The $\zeta_{0}$ determines the resonant bandwidth of the cavity. In a typical experiment with a low loss $\chi^{(2)}$-crystal [3] $\gamma_{T} \sim 57 \mathrm{MHz}, \gamma_{L} \sim 1.2 \mathrm{MHz}$, and $\zeta_{0} / \pi \sim 9.3 \mathrm{MHz}$ (the full width at half maximum).

A small fraction is taken from the squeezed state and is split into paths $\mathrm{B}$ and $\mathrm{C}$ as the trigger beams, resulting the three-beam squeezed state

$$
\hat{\rho}_{A B C}=\hat{V}_{B C} \hat{V}_{A B} \hat{\rho}_{A}^{(0)} \hat{V}_{A B}^{\dagger} \hat{V}_{B C}^{\dagger} \cdot
$$

The trigger beams are then measured by two on/off photodetectors with the time resolution $T(\sim 1 \mathrm{~ns})$. Its mathematical modeling is described in [18]. Denoting the "on"-signal elements of the positive operator-valued measure (POVM) of the on/off detectors as $\bar{\Pi}_{B}$ and $\bar{\Pi}_{C}$, the output state in path A conditioned by the "on" signals is given by

$$
\hat{\rho}_{A}=\frac{1}{P_{d e t}} \operatorname{Tr}_{B C}\left(\hat{\rho}_{A B C} \bar{\Pi}_{B} \otimes \bar{\Pi}_{C}\right)
$$

where

$$
P_{\text {det }}=\operatorname{Tr}_{A B C}\left(\hat{\rho}_{A B C} \bar{\Pi}_{B} \otimes \bar{\Pi}_{C}\right)
$$

is the detection probability.

This state is still a cw beam, consisting of multimodes. We are, however, interested in the two modes; the unbiased modes $\left\{\Psi_{U 1}(t), \Psi_{U 2}(t)\right\}$ and the biased modes $\left\{\Psi_{+}(t), \Psi_{-}(t)\right\}$. Denoting these sets as $\left\{\Psi_{k}(t)\right\}$ we introduce the annihilation operators

$$
\hat{A}_{k} \equiv \int_{-\infty}^{\infty} d t \hat{A}(t) \Psi_{k}(t)
$$

Let the quadrature amplitude and phase operators be

$$
\hat{X}_{k}=\frac{\hat{A}_{k}+\hat{A}_{k}^{\dagger}}{\sqrt{2}}, \quad \hat{P}_{k}=\frac{\hat{A}_{k}-\hat{A}_{k}^{\dagger}}{\sqrt{2} i} .
$$

In the homodyne channel one can observe a reduced state from $\hat{\rho}_{A}$ into a desired mode by filtering the homodyne current into

$$
\hat{X}_{k}(\phi)=\frac{\hat{A}_{k} e^{-i \phi}+\hat{A}_{k}^{\dagger} e^{i \phi}}{\sqrt{2}}
$$




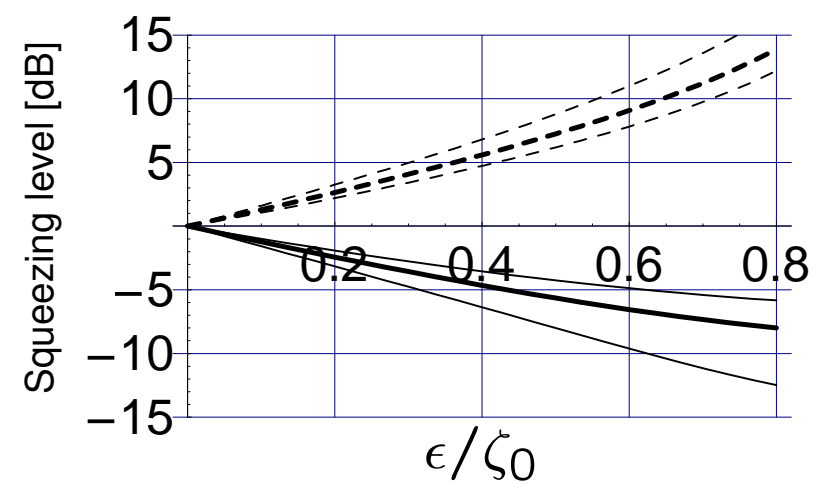

FIG. 6: Squeezing and antisqueezing characteristics of the tempral mode $\psi(t)=\sqrt{f} e^{-f|t|}$ as a function of $\epsilon / \zeta_{0}$. The solid (dashed) lines are for $f=10,30$, and $50 \mathrm{MHz}$, from the bottom (top).

and by constructing the Wigner functions with respect to this quadrature amplitude.

\section{B. Numerical results}

In Fig. 6 the squeezing and antisqueezing characteristics of the input beam are shown for the tempral mode $\psi(t)=\sqrt{f} e^{-f|t|}$ for $f=10,30$, and $50 \mathrm{MHz}$. Since the squeezing itself is larger at lower frequencies, filtering with wider tempral shape observes larger squeezing. But the photon-subtracted squeezed state in the cw scheme is most appropriately observed for a characteristic frequency of the OPO $f \sim \zeta_{0} \sim 30 \mathrm{MHz}$ (the thick solid and dashed lines in Fig. 6). The reason for it includes a fact that the photon counting by Si APD takes place in a time scale much shorter than the squeezing correlation time.

Figure 7 shows the Wigner functions (see Appendix for the formulae) and their contour plots; $a$ is for the biased mode $\Psi_{+}(t)$, b is for the biased mode $\Psi_{-}(t)$, and $\mathrm{c}$ is for the unbiased mode $\Psi_{U 1}(t)$. The nonlinear coefficient is taken as $\epsilon / \zeta_{0}=0.3$, corresponding to $-3.6 \mathrm{~dB}$ squeezing for the localized mode $\psi(t)=\sqrt{\zeta}_{0} e^{-\zeta_{0}|t|}$. The reflectance of the tapping BS is set to $R=0.05$ (i.e. $5 \%$ ). The quantum efficiency of the homodyne detector is taken as $\eta_{H}=0.96 \mathrm{in} \mathrm{Eq}$. (A38). For the on/off detectors, the overall detection efficiency of $\eta=0.6$ and the fake trigger rate of $\nu=10^{-7}$ are assumed in Eq. A23. Since the Wigner functions are identical for both of the unbiased modes, only the ones for $\Psi_{U 1}(t)$ are shown. From left to right $\Delta=1,30,50$, and $250 \mathrm{~ns}$. Here note that the original two-mode state is essentially an entangled state. So a reduced state to a particular mode is more or less a mixed state. But one can see typical features of kitten components behind.

The left two columns are typical cases of $\zeta_{0} \Delta \lesssim 1$. For $\Delta=1 \mathrm{~ns}$ an even-number kitten is generated in mode
$\Psi_{+}(t)$, while a nearly vacuum state is in mode $\Psi_{-}(t)$. The reduced state to mode $\Psi_{U 1}(t)$ seems close to a thermalized squeezed vacuum. For $\Delta=30 \mathrm{~ns}$ an amplified even-number kitten appears in mode $\Psi_{+}(t)$ with a deeper negative Wigner function. The reduced state to mode $\Psi_{U 1}(t)$ becomes close to a mixture of two Gaussian states.

The right two columns are typical cases of $\zeta_{0} \Delta>1$. For $\Delta=50 \mathrm{~ns}$ the reduced state to mode $\Psi_{+}(t)$ still has a negative Wigner function, and larger amplitude. The state seen in mode $\Psi_{U 1}(t)$ shows a dip at the phase space origin, which is a typical feature of odd-number kitten. Actually $W(0,0)$ directly reflects the parity of dominant photon number, namely a dip for odd and peak for even. For $\Delta=250 \mathrm{~ns}$ one has two odd-number kittens as seen in mode $\Psi_{U 1}(t)$. In terms of the biased modes $\Psi_{ \pm}(t)$ the states reduces to identical mixed states of the evennumber cat and the squeezed vacuum.

Figure 8 shows the case of $\Delta=65 \mathrm{~ns}$. The upper panels $\mathrm{a}$ and $\mathrm{b}$ show the Wigner functions and their contour plots of the states reduced to the unbiased modes, while the lower panels $\mathrm{c}$ and $\mathrm{d}$ show the ones of the states reduced to the biased modes. For this time separation the reduced states in both kinds of modes show the negative Wigner functions. The panels a and $\mathrm{b}$ correspond to the odd-number kittens, while the pannel c and d correspond to the squeezed vacuum and the even-number cat. (These reduced states are more or less mixed ones due to the two-mode entanglement behind.) Thus by changing the filtering functions one can access different features of the components. One possible way of viewing the change from $\mathrm{a}$ and $\mathrm{b}$ to $\mathrm{c}$ and $\mathrm{d}$ in Fig. 8 is a constructive bosonic interference of the mesoscopic superposition states in the sense that odd-number-rich components seen in modes $\Psi_{U 1}(t)$ and $\Psi_{U 2}(t)$ condensate into mode $\Psi_{+}(t)$ constructively, producing an amplified even-number-rich cat. On the other hand, in mode $\Psi_{-}(t)$ the destructive interference occurs, leaving the vacuum-like state.

In Fig. 9 we depict average photon numbers as a function of $\Delta$ for five kinds of squeezing levels, from the bottom $\epsilon / \zeta_{0}=0.1(-1.2 \mathrm{~dB}), 0.2(-2.4 \mathrm{~dB}), 0.3(-3.6 \mathrm{~dB}), 0.4(-$ $4.7 \mathrm{~dB})$ and $0.5(-5.7 \mathrm{~dB})$. See Fig. 66 for the corresponding squeezing levels for $f=30 \mathrm{MHz}$. (The formulae are given in the last part of Appendix.) The left is for the biased modes $\Psi_{+}(t)$ (solid line) and $\Psi_{-}(t)$ (dashed line). The right is for one of the unbiased modes. Since $\left\langle\hat{n}_{1}\right\rangle=\left\langle\hat{n}_{2}\right\rangle$ for the unbiased modes, the right panel directly show how the total average photon number increases and saturates to a certain level as $\zeta_{0} \Delta$ increases.

An interesting feature is the peak structure in mode $\Psi_{+}(t)$ seen in the left panel. Most of photons accumulate in mode $\Psi_{+}(t)$ around $\zeta_{0} \Delta \sim 1 \sim 3$, depending on the squeezing levels. On the other hand, the average photon number in mode $\Psi_{-}(t)$ is suppressed in this time range. Roughly speaking amplified even-number kittens appear in this time range. A typical Wigner function for larger squeezing $\epsilon / \zeta_{0}=0.5$ with $\Delta=43 \mathrm{~ns}$ is shown in Fig. 10 This is close to a quantum superposition of 
1ns
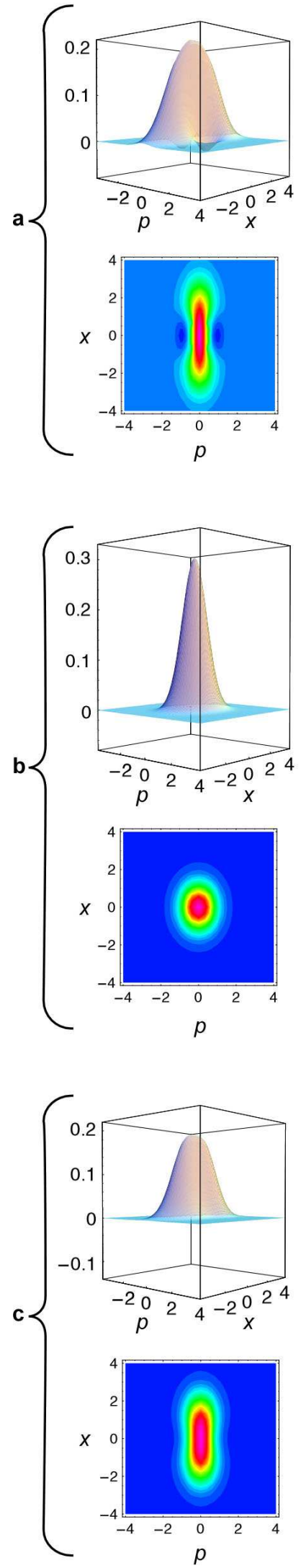

30ns
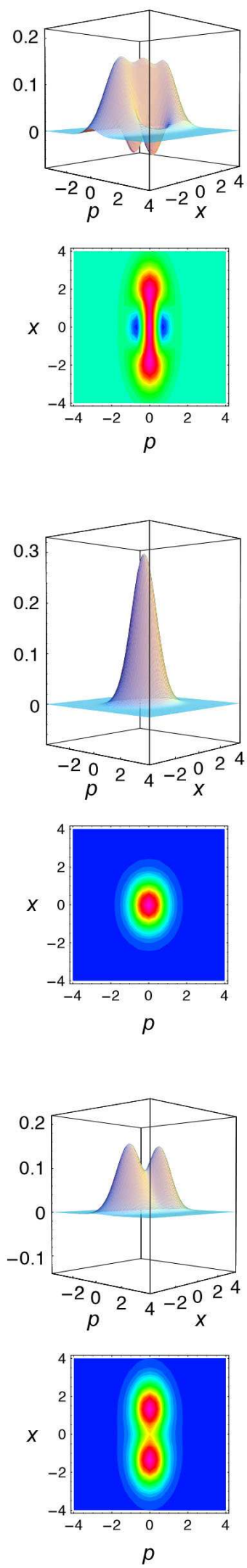

50ns
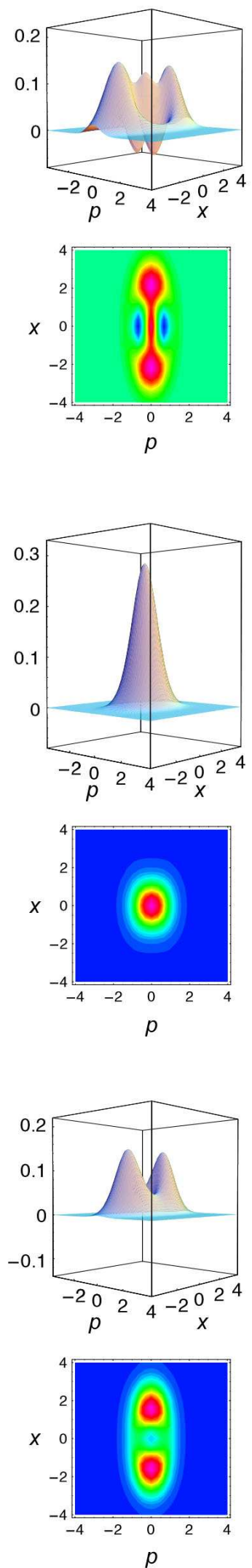
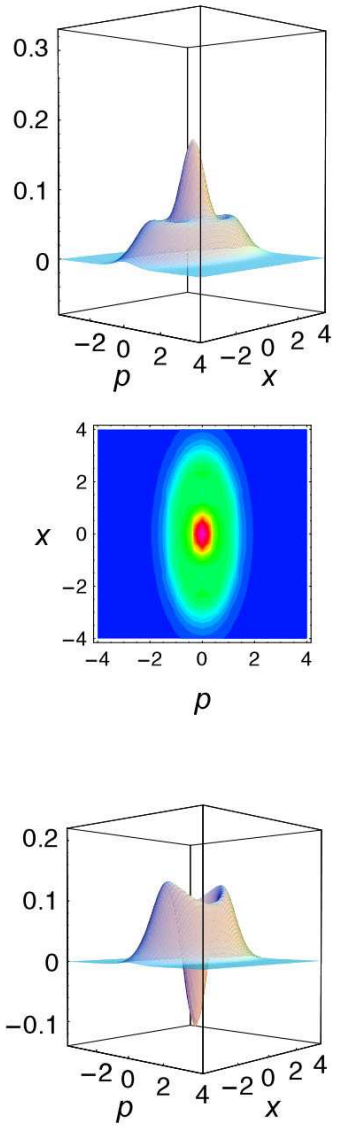

250ns
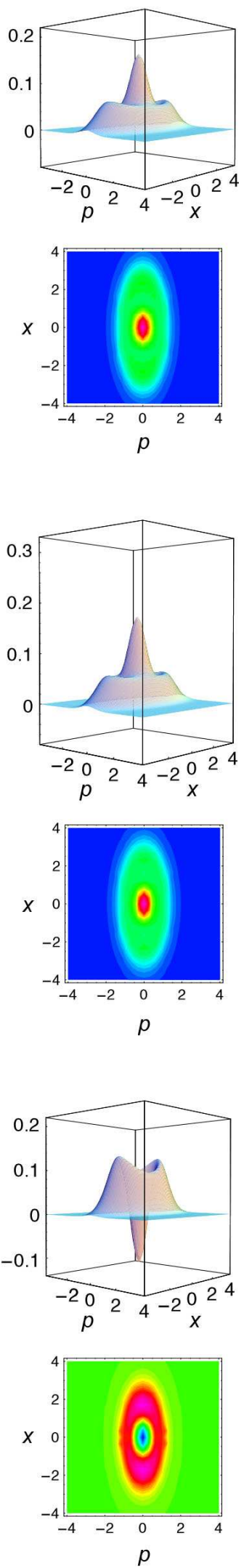

FIG. 7: Wigner functions and their contour plots. a for the biased mode $\Psi_{+}(t)$. b for the biased mode $\Psi_{-}(t)$. c for the unbiased mode $\Psi_{U 1}(t)$. From left to right $\Delta=1,30,50$, and $250 \mathrm{~ns}$. 

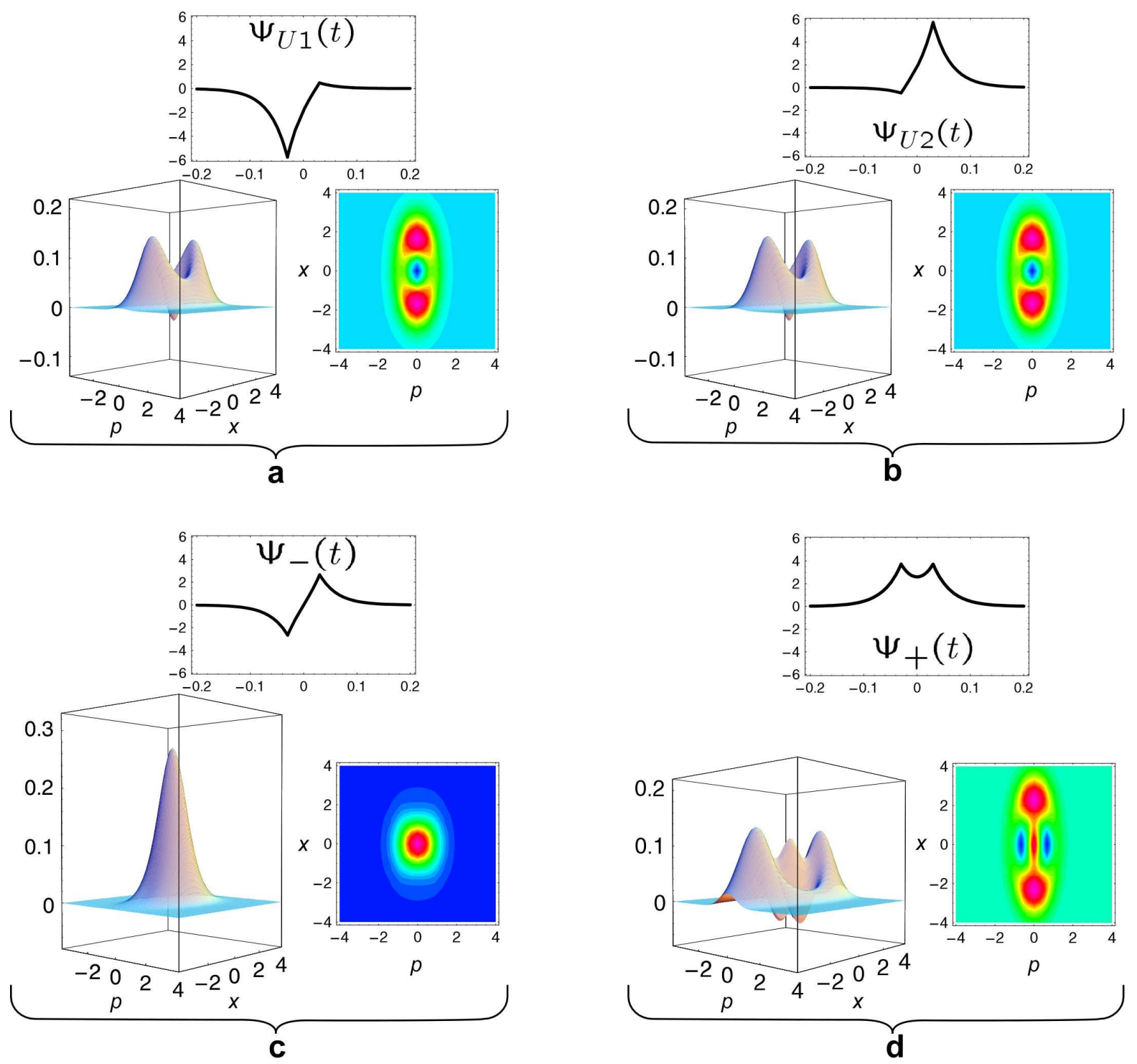

FIG. 8: Wigner functions and their contour plots for the two sets of modes in the case of $\Delta=65 \mathrm{~ns}$.
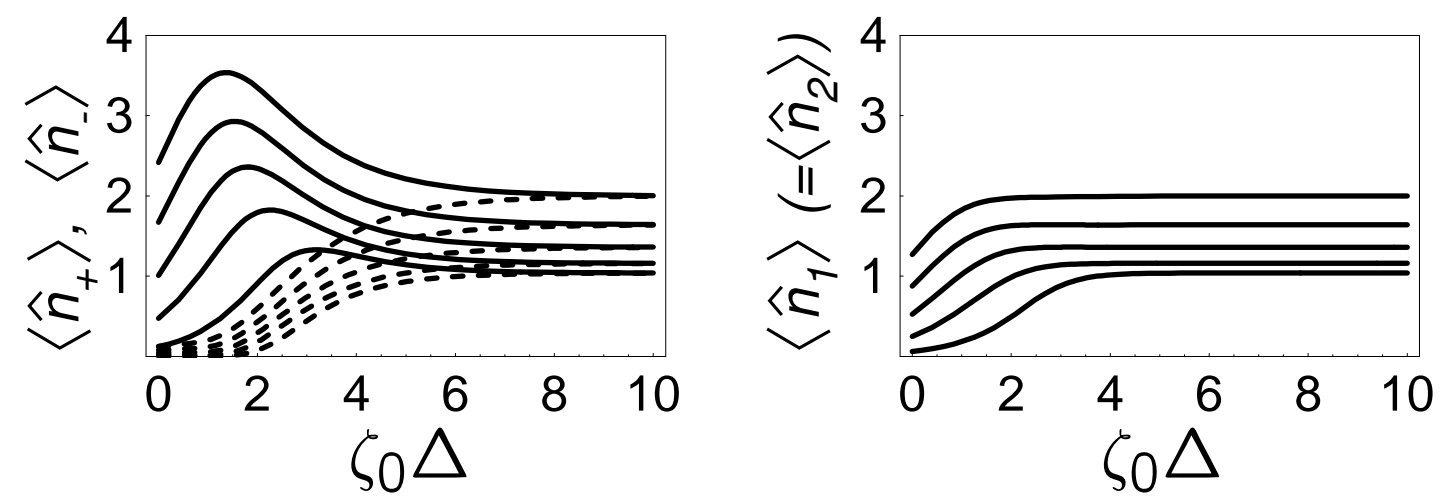

FIG. 9: Average photon numbers as a function of $\zeta_{0} \Delta$ for five kinds of squeezing levels, from the bottom $\epsilon / \zeta_{0}=0.1,0.2,0.3$, 0.4 and 0.5. The left is for the biased modes $\Psi_{+}(t)$ (solid line) and $\Psi_{-}(t)$ (dashed line). The right is for one of the unbiased modes. 


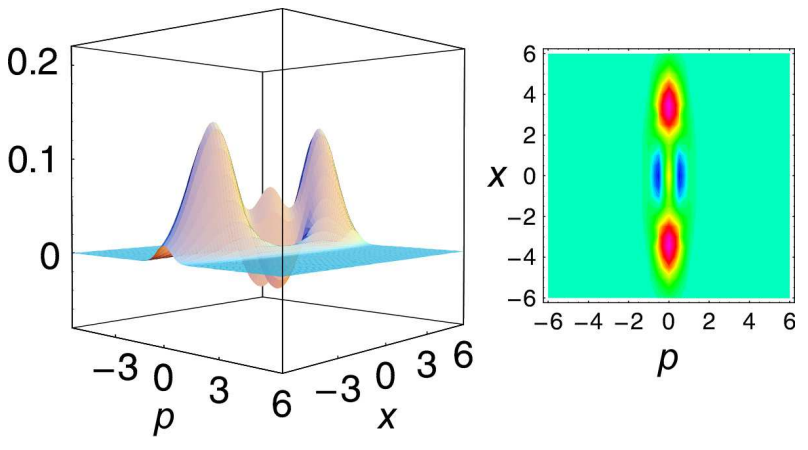

FIG. 10: Wigner function and its contour plot of the state in mode $\Psi_{+}(t)$ around the peak of $\left\langle\hat{n}_{+}\right\rangle$, corresponding to $\epsilon / \zeta_{0}=0.5$ and $\Delta=43 \mathrm{~ns}$.

distinct squeezed states with phase difference of $\pi$. Interestingly this is quite similar to the cat state generated by four-photon subtraction, which was predicted in [6]. Precisely speaking the maximum quantumness of the cats including the negativity of the Wigner function and the state purity does not necessarily coincide to the peak of $\left\langle\hat{n}_{+}\right\rangle$. The detailed analysis will be presented in another paper [19].

The process of producing the amplified kittens is nothing but the constructive bosonic interefence caused by nonlinear process of photon subtraction. This is directly seen as the superposition in Eq. (24). The mechanism behind this consists of the following two aspects. The first one is the indistingushable two-photon subtraction from the temporally overlapped squeezed packets. This aspect is represented by the term $\hat{S}_{A} \hat{A}_{2}^{\dagger} \hat{A}_{1}^{\dagger}\left|\mathbf{0}_{A}\right\rangle$ in Eq. (12), or equivalently the first and second terms in Eq. (24). Note that the two-photon subtraction leaves the state that can be represented as the squeezed two-photon states. The second one is the existence of the squeezed vacuum as the second term in Eq. (12). This corresponds to the term $\hat{S}\left|0_{+}, 0_{-}\right\rangle[=\hat{S}|0,0\rangle]$ in Eq. (24) [Eq. (19)]. This term allows one to tune the coefficients of superposition between the squeezed two-photon state and the squeezed vacuum. The second aspect makes a sharp contrast with a similar scheme with nondegenerate two-mode squeezed vacuum to generate two-photon state proposed in [15], where such a term does not exist.

The state structure with the parameters $\Delta$ and the squeezing coefficient $\epsilon$ explained above allows one to control the bosonic interference in the time domain, and generate variety of quantum superposition states.

\section{SUMMARY}

We studied non-Gaussian states generated by twophoton subtraction from a cw squeezed light source. A trigger photon click specifies a certain temporally localized mode in the remaining squeezed beam. The twophoton subtraction from overlappping squeezed pack- ets generates temporally multiplexed Schrödinger kittens with a designated time separation $\Delta$. They are generally an entangled states of even- and odd-number kittens, and the squeezed vacuum over two temporal modes. We have numerically studied these states by calculating the Wigner fuctions of the reduced states into each mode of the two kinds of sets. In the biased mode $\Psi_{+}(t)$ amplified kittens are produced in a region $\Delta \sim \zeta_{0}^{-1}$ where the time separation is comparable with the correlation time of squeezed packets. This is due to constructive bosonic interference in the time domain.

As for furure perspective, it is interesting to consider ways of combining temporally multiplexed cat states with spatial degree of freedom to implement resource-effective quantum computation, and to generate useful nonclassical resources for metrology. It is also interesting to apply the similar operation with overlapping squeezed packets to other kinds of bosonic systems in other degrees of freedom such as the spatial and/or momentum domain.

\section{Acknowledgments}

The authors acknowledge M. S. Kim for helpful discussions. This work was supported by a MEXT Grant-inAid for Scientific Research (B) 19340115, and for Young Scientists (B) 19740253.

\section{APPENDIX A: MATHEMATICAL FORMULAE FOR TWO-PHTON SUBTRACTION}

In this Appendix we present methematical formulae to analyze the two-photon subtracted squeezed states in the cw scheme.

The trigger channel consists of on/off detectors with the the time resolution $T(\sim 1 \mathrm{~ns})$. The trigger photon mode is assumed to be rectangular with the duration $T$

$$
\phi_{k}(t)= \begin{cases}\frac{1}{\sqrt{T}} \exp \left(-i \frac{2 \pi k t}{T}\right) & -\frac{T}{2} \leq t \leq \frac{T}{2}, \\ 0 & \text { otherwise }\end{cases}
$$

Since $\zeta_{0} T \ll 1$ only the lowest mode $\phi_{0}(t)$ is excited in the photodetectors, and the weights of the other higher modes are negligible [18]. The trigger fields at $t_{1}$ - and $t_{2}$-segments are defined by

$$
\begin{aligned}
\bar{B} & \equiv \frac{1}{\sqrt{T}} \int_{t_{1}-T / 2}^{t_{1}+T / 2} d t \hat{B}(t) \\
\bar{C} & \equiv \frac{1}{\sqrt{T}} \int_{t_{2}-T / 2}^{t_{2}+T / 2} d t \hat{C}(t) .
\end{aligned}
$$

Let $\bar{\Pi}_{B}$ and $\bar{\Pi}_{C}$ be the "on" signal elements of the positive operator-valued measure (POVM) of the on/off detectors given in [18]. 
The output state in path A conditioned by the "on" signals is given by

$$
\hat{\rho}_{A}=\frac{1}{P_{d e t}} \operatorname{Tr}{ }_{B C}\left(\hat{\rho}_{A B C} \bar{\Pi}_{B} \otimes \bar{\Pi}_{C}\right)
$$

where

$$
P_{\text {det }}=\operatorname{Tr}_{A B C}\left(\hat{\rho}_{A B C} \bar{\Pi}_{B} \otimes \bar{\Pi}_{C}\right)
$$

is the detection probability. Here note that we discard the information of the "off" signals.

This state is still a cw beam, consisting of multimodes. We are, however, interested in the two modes; the unbiased modes $\left\{\Psi_{U 1}(t), \Psi_{U 2}(t)\right\}$ and the biased modes $\left\{\Psi_{+}(t), \Psi_{-}(t)\right\}$. Denoting these sets as $\left\{\Psi_{k}(t)\right\}$ we introduce the annihilation operators

$$
\hat{A}_{k} \equiv \int_{-\infty}^{\infty} d t \hat{A}(t) \Psi_{k}(t)
$$

Let the quadrature amplitude and phase operators be

$$
\hat{X}_{k}=\frac{\hat{A}_{k}+\hat{A}_{k}^{\dagger}}{\sqrt{2}}, \quad \hat{P}_{k}=\frac{\hat{A}_{k}-\hat{A}_{k}^{\dagger}}{\sqrt{2} i}
$$

their vector notation be

$$
\hat{\mathbf{X}}_{A} \equiv\left(\begin{array}{c}
\hat{X}_{1} \\
\hat{X}_{2}
\end{array}\right), \quad \hat{\mathbf{P}}_{A} \equiv\left(\begin{array}{c}
\hat{P}_{1} \\
\hat{P}_{2}
\end{array}\right)
$$

and for beam B and $\mathrm{C}$ be

$$
\begin{array}{ll}
\bar{X}_{B}=\frac{\bar{B}+\bar{B}^{\dagger}}{\sqrt{2}}, & \bar{P}_{B}=\frac{\bar{B}-\bar{B}^{\dagger}}{\sqrt{2} i} \\
\bar{X}_{C}=\frac{\bar{C}+\bar{C}^{\dagger}}{\sqrt{2}}, & \bar{P}_{C}=\frac{\bar{C}-\bar{C}^{\dagger}}{\sqrt{2} i} .
\end{array}
$$

The three-beam squeezed state in the relevant modes is conveniently described by the characteristic function

$$
\begin{aligned}
& C\left(\hat{\rho}_{A B C} ; \mathbf{u}_{A}, \mathbf{v}_{A}, u_{B}, v_{B}, u_{C}, v_{C}\right) \\
& =\operatorname{Tr}{ }_{A B C}\left\{\hat { \rho } _ { A B C } \operatorname { e x p } \left[i \left({ }^{t} \mathbf{u}_{A} \hat{\mathbf{X}}_{A}+{ }^{t} \mathbf{v}_{A} \hat{\mathbf{P}}_{A}\right.\right.\right. \\
& \left.\left.\left.\quad+u_{B} \bar{X}_{B}+v_{B} \bar{P}_{B}+u_{C} \bar{X}_{C}+v_{C} \bar{P}_{C}\right)\right]\right\} \\
& =\exp \left[-\frac{1}{4}{ }^{t} \mathbf{u} \boldsymbol{\Gamma}(-\epsilon) \mathbf{u}\right] \exp \left[-\frac{1}{4}{ }^{t} \mathbf{v} \boldsymbol{\Gamma}(\epsilon) \mathbf{v}\right],
\end{aligned}
$$

where

$$
\begin{aligned}
& \mathbf{u} \equiv\left(\begin{array}{l}
\mathbf{u}_{A} \\
u_{B} \\
u_{C}
\end{array}\right), \quad \mathbf{v} \equiv\left(\begin{array}{c}
\mathbf{v}_{A} \\
v_{B} \\
v_{C}
\end{array}\right) \\
& \mathbf{u}_{A} \equiv\left(\begin{array}{l}
u_{1} \\
u_{2}
\end{array}\right), \quad \mathbf{v}_{A} \equiv\left(\begin{array}{l}
v_{1} \\
v_{2}
\end{array}\right) .
\end{aligned}
$$

The covariance matrix is given by

$$
\boldsymbol{\Gamma}(\epsilon) \equiv\left(\begin{array}{ccc}
\boldsymbol{\Gamma}_{A A}(\epsilon) & \sqrt{1-R_{1}} \boldsymbol{\xi}_{B}(\epsilon) & \sqrt{R_{1}} \boldsymbol{\xi}_{C}(\epsilon) \\
\sqrt{1-R_{1}} \boldsymbol{\xi}_{B}(\epsilon) & 1+\left(1-R_{1}\right) b_{++}(\epsilon) & \sqrt{\left(1-R_{1}\right) R_{1}} b_{+-}(\epsilon) \\
\sqrt{R_{1}} \boldsymbol{\xi}_{C}(\epsilon) & \sqrt{\left(1-R_{1}\right) R_{1}} b_{+-}(\epsilon) & 1+R_{1} b_{++}(\epsilon)
\end{array}\right)
$$

where

$$
\begin{gathered}
\boldsymbol{\Gamma}_{A A}(\epsilon) \equiv R \mathbf{I}+(1-R)\left(\begin{array}{cc}
M_{11}(\epsilon) & M_{12}(\epsilon) \\
M_{21}(\epsilon) & M_{22}(\epsilon)
\end{array}\right) \quad(\mathrm{A} 15) \\
M_{k l}(\epsilon)=\delta_{k l}-\frac{\epsilon \gamma_{T}}{\zeta(\epsilon)} \int_{-\infty}^{\infty} d t \int_{-\infty}^{\infty} d t^{\prime} \Psi_{k}(t) \Psi_{l}\left(t^{\prime}\right) e^{-\zeta(\epsilon)\left|t-t^{\prime}\right|} \\
\boldsymbol{\xi}_{B}(\epsilon) \equiv\left(\begin{array}{c}
\xi_{1}^{B}(\epsilon) \\
\xi_{2}^{B}(\epsilon)
\end{array}\right), \quad \boldsymbol{\xi}_{C}(\epsilon) \equiv\left(\begin{array}{c}
\xi_{1}^{C}(\epsilon) \\
\xi_{2}^{C}(\epsilon)
\end{array}\right) \\
\xi_{k}^{B}(\epsilon)=\sqrt{(1-R) R} \frac{\epsilon \gamma_{T}}{\zeta(\epsilon)} \sqrt{T} \int_{-\infty}^{\infty} d t \Psi_{k}(t) e^{-\zeta(\epsilon)\left|t-t_{1}\right|} \\
\xi_{k}^{C}(\epsilon)=\sqrt{(1-R) R} \frac{\epsilon \gamma_{T}}{\zeta(\epsilon)} \sqrt{T} \int_{-\infty}^{\infty} d t \Psi_{k}(t) e^{-\zeta(\epsilon)\left|t-t_{2}\right|}
\end{gathered}
$$

$$
\begin{aligned}
& b_{++}(\epsilon)=-R \frac{\epsilon \gamma_{T}}{\zeta(\epsilon)} \\
& b_{+-}(\epsilon)=b_{++}(\epsilon) e^{-\zeta(\epsilon) \Delta} .
\end{aligned}
$$

The characteristic function of the two-photon subtracted squeezed state is given by

$$
\begin{aligned}
& C\left(\hat{\rho}_{A} ; \mathbf{u}_{A}, \mathbf{v}_{A}\right) \\
& =\frac{1}{P_{d e t}} \operatorname{Tr}_{A B C}\left[\hat{\rho}_{A B C} \bar{\Pi}_{B} \otimes \bar{\Pi}_{C} e^{i\left({ }^{t} \mathbf{u}_{A} \hat{\mathbf{x}}_{A}+{ }^{t} \mathbf{v}_{A} \hat{\mathbf{P}}_{A}\right)}\right] \\
& =\frac{1}{(2 \pi)^{2} P_{\text {det }}} \int d u_{B} \int d v_{B} \int d u_{C} \int d v_{C} \\
& \quad \times C\left(\hat{\rho}_{A B C} ; \mathbf{u}_{A}, \mathbf{v}_{A}, u_{B}, v_{B}, u_{C}, v_{C}\right) \\
& \quad \times C\left(\bar{\Pi}_{B} ;-u_{B},-v_{B}\right) C\left(\bar{\Pi}_{C} ;-u_{C},-v_{C}\right) . \quad(\mathrm{A} 22)
\end{aligned}
$$

The characteristic function of the "on" signal POVM element is given by

$$
\begin{aligned}
C\left(\bar{\Pi}_{B} ; u_{B}, v_{B}\right) & =2 \pi \delta\left(u_{B}\right) \delta\left(v_{B}\right) \\
& -\frac{e^{-\nu}}{\eta} \exp \left[-\frac{2-\eta}{4 \eta}\left(u_{B}^{2}+v_{B}^{2}\right)\right]
\end{aligned}
$$


where $\eta$ is the overall detection efficiency, and $\nu$ is the fake trigger rate. Substituting it into Eq. (A22) we have

$$
\begin{aligned}
C\left(\hat{\rho}_{A} ; \mathbf{u}_{A}, \mathbf{v}_{A}\right)=\sum_{i=1}^{4} \frac{\mathcal{N}^{(i)}}{P_{d e t}} \exp \left[-\frac{{ }^{t} \mathbf{u}_{A} \boldsymbol{\Gamma}^{(i)}(-\epsilon) \mathbf{u}_{A}}{4}\right] \\
\times \exp \left[-\frac{{ }^{t} \mathbf{v}_{A} \boldsymbol{\Gamma}^{(i)}(\epsilon) \mathbf{v}_{A}}{4}\right]
\end{aligned}
$$

where

$$
\begin{aligned}
& \mathcal{N}^{(1)}=1 \\
& \mathcal{N}^{(2)}=-\frac{2 e^{-\nu}}{\eta \sqrt{b^{(2)}(-\epsilon) b^{(2)}(\epsilon)}} \\
& \mathcal{N}^{(3)}=-\frac{2 e^{-\nu}}{\eta \sqrt{b^{(3)}(-\epsilon) b^{(3)}(\epsilon)}} \\
& \mathcal{N}^{(4)}=\frac{4 e^{-2 \nu}}{\eta^{2} \sqrt{b^{(4)}(-\epsilon) b^{(4)}(\epsilon)}} \\
& b^{(1)}(\epsilon)=1 \\
& b^{(2)}(\epsilon)=\frac{2}{\eta} R_{1} b_{++}(\epsilon) \\
& b^{(3)}(\epsilon)=\frac{2}{\eta}\left(1-R_{1}\right) b_{++}(\epsilon) \\
& b^{(4)}(\epsilon)=b^{(3)}(\epsilon) b^{(2)}(\epsilon)-\left(1-R_{1}\right) R_{1} b_{+-}^{2}(\epsilon) \\
& P_{\text {det }}=\sum_{i=1}^{4} \mathcal{N}^{(i)} \\
& \Gamma_{k l}^{(i)}(\epsilon)=R+(1-R) M_{k l}(\epsilon)-\frac{j_{k l}^{(i)}(\epsilon)}{b^{(i)}(\epsilon)} \\
& j_{k l}^{(1)}(\epsilon)=0 \\
& j_{k l}^{(2)}(\epsilon)=R_{1} \xi_{k}^{C}(\epsilon) \xi_{l}^{C}(\epsilon) \\
& j_{k l}^{(3)}(\epsilon)=\left(1-R_{1}\right) \xi_{k}^{B}(\epsilon) \xi_{l}^{B}(\epsilon) \\
& j_{k l}^{(4)}(\epsilon)=R_{1} b^{(3)}(\epsilon) \xi_{k}^{C}(\epsilon) \xi_{l}^{C}(\epsilon) \\
& +\left(1-R_{1}\right) b^{(2)}(\epsilon) \xi_{k}^{B}(\epsilon) \xi_{l}^{B}(\epsilon) \\
& \text { - } 2\left(1-R_{1}\right) R_{1} b_{+-}(\epsilon) \xi_{k}^{B}(\epsilon) \xi_{l}^{C}(\epsilon)
\end{aligned}
$$

In real experiment the output state suffers from propagation loss, and imperfect efficiency of homodyne detection. Taking these factors into account by the effective transmission coefficient $\eta_{H}$ the observed state $\hat{\rho}_{A}^{\prime}$ is characterized by

$$
\begin{aligned}
C\left(\hat{\rho}_{A}^{\prime} ; \mathbf{u}_{A}, \mathbf{v}_{A}\right)=\sum_{i=1}^{4} & \frac{\mathcal{N}^{(i)}}{P_{d e t}} \exp \left[-\frac{{ }^{t} \mathbf{u}_{A} \boldsymbol{\Gamma}_{H}^{(i)}(-\epsilon) \mathbf{u}_{A}}{4}\right] \\
& \times \exp \left[-\frac{{ }^{t} \mathbf{v}_{A} \boldsymbol{\Gamma}_{H}^{(i)}(\epsilon) \mathbf{v}_{A}}{4}\right]
\end{aligned}
$$

where

$$
\boldsymbol{\Gamma}_{H}^{(i)}(\epsilon)=\left(1-\eta_{H}\right) \mathbf{I}+\eta_{H} \boldsymbol{\Gamma}^{(i)}(\epsilon)
$$

The output state also suffers from invasion of external noise photons from various parts, which degrades quantum correlations. This effect can be modeled by a mixing of thermal vacuum. Mathematically this can be taken into account by replacing the factor $\epsilon \gamma_{T} / \zeta(\epsilon)$ in Eqs. A16), A18), A19), and A20 with $\left(\epsilon-\epsilon_{x}\right) \gamma_{T} / \zeta(\epsilon)$ where $\epsilon_{x}$ represents the degree of external noise photons.

The two-mode Wigner function is then given by

$$
\begin{aligned}
W(\mathbf{x}, \mathbf{p})= & \frac{1}{(2 \pi)^{4}} \int d \mathbf{u}_{A} d \mathbf{v}_{A} C\left(\hat{\rho}_{A}^{\prime} ; \mathbf{u}_{A}, \mathbf{v}_{A}\right) e^{i\left({ }^{t} \mathbf{u}_{A} \mathbf{x}+{ }^{t} \mathbf{v}_{A} \mathbf{p}\right)} \\
=\sum_{i=1}^{4} \frac{\mathcal{N}^{(i)}}{\pi^{2} P_{d e t}} & \frac{\exp \left[-{ }^{t} \mathbf{x} \boldsymbol{\Gamma}_{H}^{(i)}(-\epsilon)^{-1} \mathbf{x}\right]}{\sqrt{\left|\boldsymbol{\Gamma}_{H}^{(i)}(-\epsilon)\right|}} \\
& \times \frac{\exp \left[-{ }^{t} \mathbf{p} \boldsymbol{\Gamma}_{H}^{(i)}(\epsilon)^{-1} \mathbf{p}\right]}{\sqrt{\left|\boldsymbol{\Gamma}_{H}^{(i)}(\epsilon)\right|}} .
\end{aligned}
$$

It is hard to depict the two-mode Wigner function numerically in a graph. One way to understand the state properties is to see the Wigner function reduced to an appropriate single mode $\Psi_{k}(t)(k=U 1, U 2,+$, or - $)$. It is given by

$$
W(x, p)=\sum_{i=1}^{4} \frac{\mathcal{N}^{(i)} \exp \left[-\frac{x^{2}}{\Gamma_{H k k}^{(i)}(-\epsilon)}-\frac{p^{2}}{\Gamma_{H k k}^{(i)}(\epsilon)}\right]}{\pi P_{\operatorname{det}} \sqrt{\Gamma_{H k k}^{(i)}(-\epsilon) \Gamma_{H k k}^{(i)}(\epsilon)}} .
$$

The photon number in each mode can be calculated by the characteristic function by

$$
\begin{aligned}
\left\langle\hat{n}_{k}\right\rangle & =\left\langle\hat{A}_{k}^{\dagger} \hat{A}_{k}\right\rangle \\
& =-\frac{1}{2}\left[\left(\frac{\partial^{2}}{\partial u_{k}^{2}}+\frac{\partial^{2}}{\partial v_{k}^{2}}\right) C\left(\hat{\rho}_{A} ; \mathbf{u}_{A}, \mathbf{v}_{A}\right)\right]_{\mathbf{u}_{A}=\mathbf{v}_{A}=0} \\
& -\frac{1}{2} \\
& =\frac{1}{4 P_{d e t}} \sum_{i=1}^{4} \mathcal{N}^{(i)}\left[\Gamma_{k k}^{(i)}(-\epsilon)+\Gamma_{k k}^{(i)}(\epsilon)\right] \\
& -\frac{1}{2}
\end{aligned}
$$

This can be reduced to explicit expressions for small $\zeta_{0} T \ll 1$ as

$$
\left\langle\hat{n}_{ \pm}\right\rangle=\frac{z^{4} G_{1}^{( \pm)}(\Delta)+z^{2} G_{2}^{( \pm)}(\Delta)}{2\left(1 \pm I_{\Delta}\right)\left[z^{2}\left(1+I_{\Delta}^{2}\right)+e^{-2 \Delta}\right]}
$$

where $z \equiv \zeta_{0} / \epsilon$

$$
\begin{aligned}
G_{1}^{( \pm)}(\Delta) \equiv & \left(1+I_{\Delta}^{2}\right) g_{\Delta}^{( \pm)}+2\left(1 \pm I_{\Delta}\right) f_{\Delta}^{( \pm) 2} \\
G_{2}^{( \pm)}(\Delta) \equiv & \psi(\Delta)^{2} g_{\Delta}^{( \pm)}+2\left(1 \pm I_{\Delta}\right)^{3} \\
& \pm 4\left(1 \pm I_{\Delta}\right) \psi(\Delta) f_{\Delta}^{( \pm) 2}
\end{aligned}
$$


with

$$
\begin{aligned}
& f_{\Delta}^{( \pm)} \equiv \frac{3}{2}\left(1 \pm I_{\Delta}\right) \pm \frac{\Delta^{2}}{2} e^{-\Delta} \\
& g_{\Delta}^{( \pm)} \equiv 5\left(1 \pm I_{\Delta}\right) \pm e^{-\Delta}\left(2 \Delta^{2}+\frac{\Delta^{3}}{3}\right)
\end{aligned}
$$

For the unbiased modes

$$
\left\langle\hat{n}_{U 1}\right\rangle=\left\langle\hat{n}_{U 2}\right\rangle
$$

[1] A. Ourjoumtsev, R. Tualle-Brouri, J. Laurat, and P. Grangier, Science, 312, 83 (2006).

[2] J. S. Neergaard-Nielsen, B. Melholt Nielsen, C. Hettich, K. Mølmer, and E. S. Polzik, Phys. Rev. Lett. 97, 083604 (2006).

[3] K. Wakui, H. Takahashi, A. Furusawa, and M. Sasaki, Opt. Express 15, 3568 (2007).

[4] A. Ourjoumtsev, R. Tualle-Brouri, and P. Grangier, Phys. Rev. Lett. 96, 213601 (2006).

[5] A. Ourjoumtsev, A. Dantan, R. Tualle-Brouri, and P. Grangier, Phys. Rev. Lett. 98, 030502 (2007).

[6] M. Dakna, T. Anhut, T. Opatrný, L. Knöll, and D.G. Welsch, Phys. Rev. A 55, 3184 (1997).

[7] T. C. Ralph, A. Gilchrist, G. J. Milburn, W. J. Munro and S. Glancy, Phys. Rev. A 68, 042319 (2003).

[8] S. J. van Enk and O. Hirota, Phys. Rev. A, 64, 022313 (2001).

[9] A. Gilchrist, K. Nemoto, W. J. Munro, T. C. Ralph, S. Glancy, S. L. Braunstein, and G. J. Milburn J. Opt. B:

$$
=\frac{1}{2}\left[\left\langle\hat{n}_{+}\right\rangle+\left\langle\hat{n}_{-}\right\rangle\right]
$$

Qunatum Semiclass. Opt6, S828 (2004).

[10] A. E. B. Nielsen and K. Mølmer, Phys. Rev. A, 76, 043840 (2007).

[11] A. P. Lund, H. Jeong, T. C. Ralph, and M. S. Kim, Phys. Rev. A, 70, 020101 (2004).

[12] A. Ourjoumtsev, H. Jeong, R. Tualle-Brouri, and P. Grangier, Nature (London) 448, 784 (2007).

[13] K. Mølmer, Phys. Rev. A 73, 063804 (2006).

[14] A. E. B. Nielsen and K. Mølmer, Phys. Rev. A 75, 023806 (2007).

[15] A. E. B. Nielsen and K. Mølmer, Phys. Rev. A 75, 043801 (2007).

[16] S. Suzuki, K. Tsujino, F. Kannari, and M. Sasaki, Opt. Commun. 259, 758 (2006).

[17] P. D. Drummond and M. D. Reid, Phys. Rev. A 41, 3930 (1990).

[18] M. Sasaki and S. Suzuki, Phys. Rev. A 73, 043807 (2006).

[19] M. Takeoka, H. Takahashi and M. Sasaki, submitted. 